\title{
Comparison of Two Commercially Available Vancomycin Discs for Interpreting Susceptibility Patterns among Gram Positive Cocci: Ongoing Challenging Journey in Resource Poor Settings
}

\author{
T. M. Nandan ${ }^{1 *}$, Abhinandnan T. Kalghatgi ${ }^{1}$, G. Latha ${ }^{2}$ and C. N. Veena $^{3}$ \\ ${ }^{1}$ Department of Microbiology, Sambhram Institute of Medical Sciences and Research, K.G.F, \\ Karnataka, India \\ ${ }^{2}$ Department of Microbiology, PES Institute of Medical sciences and Research, Kuppam, \\ Andhra Pradesh, India \\ ${ }^{3}$ Department of Physiology, Dr Chandramma Dayanand Sagar Institute of Medical Education \\ and Research, Harohalli, Karnataka, India \\ *Corresponding author
}

\section{A B S T R A C T}

\section{Keywords}

Vancomycin, disc diffusion test, comparison of commercially available discs

\section{Article Info}

Accepted:

15 December 2019 Available Online: 20 January 2020
Antibiotic discs made by different manufacturers may vary in their working potencies for various reasons and this can affect implementing standard interpretative criteria for reporting susceptibility patterns for certain key antibiotics. The present study was aimed at finding out whether zone diameters produced by certain important Gram positive cocci using two makes of vancomycin discs interfere with interpretation of susceptibility patterns and thus clinical management of patients affected when tested by the widely followed Kirby Bauer disc diffusion method in routine Microbiology practice in the scenario of resource poor settings. A random sample of 37 clinical isolates of Gram positive cocci including staphylococci, enterococci and streptococci was tested for susceptibility to vancomycin by Kirby Bauer disc diffusion method using discs of two makes and the effect of available standard breakpoint interpretative criteria for reporting susceptibility was analyzed. The average vancomycin zone diameter for the make A $(17.972 \mathrm{~mm})$ was higher than the average zone diameter for the make B $(16.013 \mathrm{~mm})$ and the difference was significant at $\mathrm{p}=0.0004$ by student $\mathrm{t}$ test. The overall variation in decisive interpretations for the isolates for the two makes was $28 \%$, more variant for enterococci $(50 \%)$ than staphylococci $(15 \%)$. Appropriate makes of vancomycin discs must be chosen as part of robust internal quality control mechanisms to minimize errors and dilemmas in interpretation of susceptibility patterns as per standard guidelines against commonly encountered gram positive cocci. All diagnostic Microbiology labs must adapt themselves to the growing need for standardization for providing better healthcare in the community.

\section{Introduction}

Same antibiotic discs made by different manufacturers vary in their working potencies for various reasons (Brown DFJ et al., 1975) and this can affect applying standard interpretative criteria (CLSI, 2010) for routinely reporting susceptibility patterns for 
certain of such antibiotic-organism combinations like staphylococci-vancomycin, enterococci-vancomycin, etc., potential superbugs, especially in resource poor healthcare laboratory settings. So, it is obvious that the management of diverse variety of diseases caused by such bacteria whose antibiotic susceptibility patterns have been interpreted thus is affected likewise. The study was inspired by a discrepancy in zone diameter that was noted during a routine internal quality control procedure using the standard strains when an additional different brand of vancomycin disc was used.

In an assessment study comparing some of major brands of antibiotic discs, Brown DFJ et al., 1975, have listed various factors that contribute towards differences in zone diameters obtained for commonly tested antibiotics including the surprising inevitability of deviation from optimal performances with discs and their contents. The causes for substandard disc quality at the end point of usage may arise right from the manufacturing process, their packing, transit and storage temperatures, humidity, etc. Further, the study had reviewed that the permitted statutory mandate in disc content to be $67-150 \%$ by FDA in the USA and that at least 6 manufacturers had not met the required target in up to a wobbling $44-100 \%$ of the discs that were produced. The scenario has not been too different in India. The three popular brands of antibiotic discs tested for conformity to recommended guidelines with respect to the zone diameters of standard strains supposed to fall within a range, Joshi A et al., 2008, found significant errors to the tune of $46.15 \%, 26 \%$, and $24 \%$ by the brands $\mathrm{X}, \mathrm{Y}$, and $\mathrm{Z}$ respectively.

\section{Aims and Objectives}

The present cross-sectional observational study on a random sample was aimed at finding out whether standard reference breakpoint diameters for two similar vancomycin discs of different makes affect interpretation of susceptibility patterns against Gram positive cocci by the widely followed Kirby Bauer disc diffusion test. It also aims at discussing possible erroneous deviations in routine reporting process in resource poor settings at the backdrop of nonavailability/impracticality of current interpretative guidelines in the interest of emphasizing the need for standardization and innovation.

\section{Materials and Methods}

The study was conducted in a rural tertiary healthcare centre in Andhra Pradesh during February to April 2014. Ethical clearance was obtained from the institutional research and ethical committee. A sample of 37 Gram positive cocci isolated from various clinical samples was tested. The isolates included staphylococci, enterococci and streptococci and were identified by standard methods like colony morphologies on sheep blood agar, Gram staining, catalase test, coagulase test, and growth characteristics on bile-esculin agar slants. Antibiotic susceptibilities to relevant indicator antibiotics were tested for identifying MRSAs, MRCONSs and $S$. pyogenes under standard recommended duration and incubation temperature. Enterococci were identified up to the generic level. All the isolates were tested for susceptibility to vancomycin, $30 \mu \mathrm{g}$ disc, by Kirby Bauer disc diffusion method (K Satyanarayana. 2009, Nandan TM et al., 2012) using discs of two makes, Make A and Make B (commercial brand identities have been kept confidential), and their zone diameters and the effects on applying standard breakpoint interpretative criteria for determination of susceptibility were analyzed. Both the brands of discs were checked for adequacy of source details, batch numbers, dates of manufacture 
and expiry. Unsupplemented Mueller-Hinton agar or sheep blood agar were used for antibiotic susceptibility testing for the nonfastidious and fastidious organisms respectively. ATCC Staphylococcus aureus 25923 was used for quality control. Suitable available Clinical Laboratory Standards Institute susceptibility testing interpretative criteria (CLSI; 2010, CLSI; 2006) were referred to for the various isolates. In view of the rampant current common practice, previous reference values were used for staphylococci as the current guidelines (CLSI, 2010 and further...) no longer recommend disc diffusion method for vancomycin susceptibility testing. However, current standard guidelines were followed for enterococci and streptococci. Zone diameter of $\geq 15 \mathrm{~mm}$ for staphylococci and $\geq 17 \mathrm{~mm}$ for enterococci and streptococci were interpreted as susceptible. Significance of variation in zone diameters for the two makes for the isolates were calculated by student' $t$ ' test and analyzed. The percentage variations in decisive interpretations (either susceptible or not) were noted for all the isolates. Other relevant classes of antibiotics that were tested as part the routine diagnostic procedure for the isolates were reported but not included in the present study.

\section{Results and Discussion}

The types and number of various clinical samples from which the isolates were derived are listed in Table 1. Pus and urine samples were a majority representing the clinical significance of staphylococci and enterococci respectively.

Table 2 lists the isolates and the zone diameters obtained using the two brands of vancomycin discs tested. Majority of the isolates were of staphylococci followed by enterococci, and streptococci. There were $S$. aureus, MRSA, and MRCONS among the staphylococci. Streptococci were of the $\alpha$ - and the $\beta$-hemolytic types. Figure1 shows the illustration in picture of the observed difference in the zone diameters using the two makes of the vancomycin discs.

The average vancomycin zone diameter for make $\mathrm{A}(17.972 \mathrm{~mm})$ was higher than the average zone diameter for make $\mathrm{B}(16.013 \mathrm{~mm})$, as depicted in Chart 1 , and the difference was significant at $\mathrm{p}=0.0004$ by student $\mathrm{t}$ test. The control strain also showed a difference of $2 \mathrm{~mm}$ between the zone diameters of the two discs.

Variation in the decisive interpretations for the major organisms, staphylococci and enterococci, wherein sensitivity/resistance was decided up on is highlighted in Chart 2. The overall variation for the isolates for the two makes was $28 \%$, more variant for enterococci $(50 \%)$ than staphylococci $(15 \%)$.

The present study attempts to bring to the fore the current ongoing challenges of the usage of vancomycin discs in susceptibility testing pertaining to commonly encountered gram positive bacteria, especially when additional variables are introduced in the form of variations in disc content.

A few similar studies (A Joshi et al., 2008) in the distant as well as in the recent past have documented variations among discs of different makes in India and elsewhere. In developing economies including in India, such discrepancies often go unnoticed and are attributable to assumptions and presumptions of sorts which are again probably due to limitations and inadequacies of internal quality control systems especially with respect to their prompt implementation. The everupdating more recent interpretative standards for different organisms and antibiotics pose additional performance and interpretative dilemmas in routine reporting processes and 
this has been the case throughout the world nations while in their continual transitions of quality improvement.

In the present study, the make B consistently showed lesser zones of diameters for the isolates than the make $\mathrm{A}$ and hence introduced a greater degree of error including being greatly affected by the standard breakpoint reference criteria for routine reporting of susceptibility of isolates to vancomycin. The study by Joshi A et al., 2008 found the 3 popular commercial discs $\mathrm{X}, \mathrm{Y}$ and $\mathrm{Z}$ to be showing discordance in interpretations of susceptibilities to the tune of $2.7 \%, 2.4 \%$, and none, respectively, when tested for major classes of antibiotics that were routinely used, but it did not include vancomycin. It is very important to note that the absence of breakpoint zone diameters for staphylococci in the current reference standards results in additional erroneous reporting incidents if the untrained interpreters use the criteria that are printed for enterococci and streptococci, viz., taking $\geq 17 \mathrm{~mm}$ as cut-off for susceptibility for all gram positive cocci instead of $\geq 15 \mathrm{~mm}$ for staphylococci, which is not being printed of late due to the proscription. This would dramatically increase the discordance level, much greater than that is found for staphylococci in this study for sheer nonavailability of any standard reference guideline!

\section{Improvement is transitional}

Although the present study apparently deviates from the current recommended guidelines, it tries to highlight the transition when realization of the need percolates downstream. Implementing updated standard guidelines for interpretation of susceptibility patterns has been a challenge worldwide and is more evident in developing economies now due to a need for adaptation to the recently brought in paradigm shift towards responsible use of antibiotics after decades of sub-standard use leading to their misuse and abuse. The Journal of Antimicrobial Chemotherapy celebrating its 40 years of publishing in Microbiology and Infectious Diseases has listed out variation from standard methods and guidelines in the performance and interpretation of antibiotic susceptibility testing as a common phenomenon that needed streamlining, albeit mentioning that much has improved over a period of time, especially with the advent of the EUCAST guidelines (M Wootton et al., 2016). Over half (50\%) of the New York State Laboratories did not comply with the then recommended NCCLS (currently CLSI) standards even for the problem organisms (staph and enterococci) in terms of their clinical burden let alone other fastidious organisms as found by a state funded study in the USA (JA Kiehlbauch et al., 2000). It resulted in a massive educational drive that provided extensive informational and feedback inputs to correct those wrong suboptimal implementation of standards. A European study (FM MacKenzie, et al., 2006) to examine adherence of various clinical laboratories to standard guidelines like CLSI found considerable deviations from the claimed procedural norms, both for the detection of VRSA and VRE; at that time, by the disc diffusion test itself!

\section{Rampant use, inevitability, and challenges for use of vancomycin disc diffusion testing}

Vancomycin susceptibility testing for staphylococci by disc diffusion method continues to be used in developing countries, currently, in spite of its proscription by the standard setting bodies including the CLSI and EUCAST (HM Naimi et al., 2017). The study recorded $100 \%$ susceptibility to vancomycin $30 \mu \mathrm{g}$ disc. Till recently, disc diffusion method was recommended for vancomycin susceptibility testing even for staphylococci along with enterococci by the national 
academia and the same has been lingering longer with the majority of the local labs following the older breakpoint criteria ( $\mathrm{K}$ Satyanarayana. 2009). Moreover, most of the standalone laboratories appear to have been out of academic contact with the resultant lag in alignment. Cost of MIC testing has been a big challenge to even majority of the urban tertiary care hospitals. Increasing number of reports have been expressing their concern regarding the issue of affordability of the available standards for interpreting the antibiotic zone diameters and the methods recommended for analyses (TP Cusack., et al., 2019, Ali K et al., 2016). Although various alternate international bodies exist to provide breakpoint diameters, they vary in their procedural methods. This adds on to the existing dilemma among their clients in the rest of the world. In an Iranian study on coagulase negative staphylococcal isolates vancomycin susceptibilities comparing disc diffusion and E-Test MIC methods, significant correlation was noted and hence routine testing was reported to be useful in resource poor healthcare settings (MTH Ashtiani et al., 2008). A similar result was obtained for staphylococci (both coagulase positive and negative) in an urban tertiary care centre in Bengaluru, India downplaying the immediate need then given the negligible prevalence of vancomycin resistance (Nandan TM et al., 2012). A multi-centre prospective surveillance study on clinical MRSA isolates in four teaching hospitals in Lahore, Pakistan, found $100 \%$ concordance between the disc diffusion and MIC broth dilution tests for susceptibility to vancomycin (SZ Bukhari et al., 2011). About $20 \%$ resistance to vancomycin among various clinical isolates of staphylococci by the disc diffusion method was reported by an Egyptian university hospital study. An older breakpoint reference diameter was used, although they do not match with the 2016 CLSI guidelines! "The isolate was considered resistant when the inhibition zone diameter (IZ) $\leq 10 \mathrm{~mm}$; intermediate when IZ from 11 to $13 \mathrm{~mm}$ and sensitive when the IZ $\geq 14$ " (ElSaid El-Banna T et al., 2018). The current standard method for hVISA detection is the disc diffusion method using a $20 \mu \mathrm{g}$ vancomycin disc and bacterial inoculum of 1.0 McFarland on BHIA supplemented with $2 \%$ $\mathrm{NaCl}$ coupled with a population analysis profile with area under the curve (PAPAUC)testing (S Wongthong et al., 2015). However, this method was reported to be complicated, expensive, and time consuming.

Furthermore, the use of standard concentration of bacterial suspension (0.5 McFarland) according to the CLSI broth and agar dilution methods have also been reported to be less sensitive in detecting the resistant subpopulation of hVISA strains. Comparing disc diffusion and agar dilution methods for detection of vancomycin susceptibility among clinical MRSA strains, a study (Sharma P et al., 2012) found the latter to be of significance in detecting VISAs but did not find heterogeneous VISAs or VRSAs. Inevitably, the authors appear to have used previously available breakpoint diameters for the disc diffusion test.

\section{Pockets of change}

However, few centres for PG studies and research have been exceptional in adhering to global advancements in testing susceptibility to vancomycin by the breakpoint MIC method (V Thati et al., 2011) and screening agar dilution method ( $\mathrm{P}$ Bora et al., 2018, R Adhikari et al., 2017). Well informed and those healthcare centres with no financial constraints are adhering to the globally recommended standards (Maj P Bhatt et al., 2015). Others should follow suit or come out with cheaper alternatives by innovative research. 
Table.1 Types and number of clinical samples

\begin{tabular}{|c|c|}
\hline Sample type & Number \\
\hline Cervical swab & 2 \\
\hline CSF & 1 \\
\hline ET secretion & 1 \\
\hline Pus & 21 \\
\hline Sputum & 2 \\
\hline Urine & 7 \\
\hline Vaginal swab & 3 \\
\hline Total & $\mathbf{3 7}$ \\
\hline
\end{tabular}

Fig.1 Disc diffusion test with discs of make ' $A$ ' and ' $B$ '

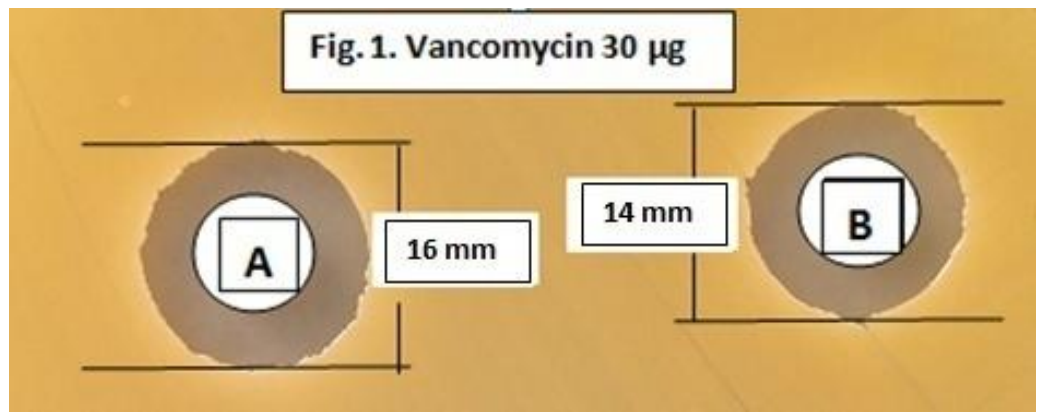

Chart.1 Mean zone diameters in millimeters

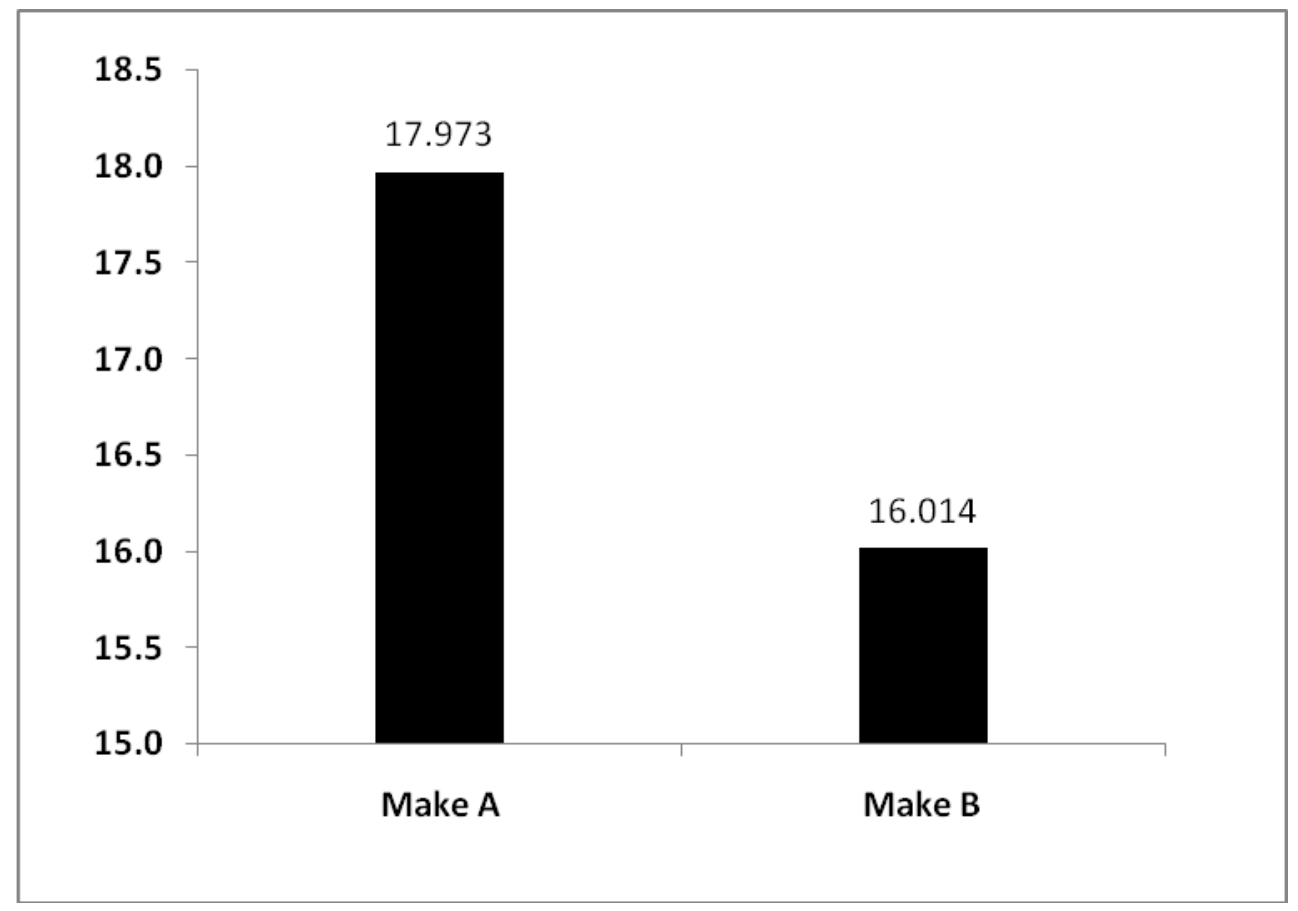


Table.2 The isolates and the zones obtained by the two brands of vancomycin discs tested

\begin{tabular}{|c|c|c|c|}
\hline \multirow[t]{2}{*}{ SI No } & \multirow[t]{2}{*}{ Isolates } & \multicolumn{2}{|c|}{$\begin{array}{l}\text { Vancomycin zone diameters in } \\
\mathrm{mm}\end{array}$} \\
\hline & & Brand A & Brand B \\
\hline (QC) & $\begin{array}{l}\text { (ATCC Staphylococcus aureus } \\
25923 \text { ) }\end{array}$ & 19 & 17 \\
\hline 1 & Beta hemolytic Streptococcus spp & 20 & 17 \\
\hline 2 & Beta hemolytic Streptococcus spp & 21 & 18 \\
\hline 3 & Enterococcus spp & 14 & 11.5 \\
\hline 4 & Enterococcus $\mathrm{spp}$ & 19 & 16 \\
\hline 5 & Enterococcus $\mathrm{spp}$ & 17 & 15 \\
\hline 6 & Enterococcus $\mathrm{spp}$ & 16.5 & 15 \\
\hline 7 & Enterococcus $\mathrm{spp}$ & 17.5 & 14.5 \\
\hline 8 & Enterococcus $\mathrm{spp}$ & 24 & 20 \\
\hline 9 & Enterococcus $\mathrm{spp}$ & 17 & 15 \\
\hline 10 & Enterococcus spp & 20 & 18 \\
\hline 11 & Enterococcus spp & 15 & 13 \\
\hline 12 & Enterococcus $\mathrm{spp}$ & 18 & 15.5 \\
\hline 13 & Enterococcus spp & 18.5 & 17.5 \\
\hline 14 & Enterococcus spp & 18 & 16 \\
\hline 15 & MRCONS & 17 & 16 \\
\hline 16 & MRCONS & 16 & 15 \\
\hline 17 & MRCONS & 22 & 20 \\
\hline 18 & MRCONS & 20 & 18 \\
\hline 19 & MRCONS & 18 & 16 \\
\hline 20 & MRSA & 17 & 14.5 \\
\hline 21 & MRSA & 15 & 14 \\
\hline 22 & MRSA & 16 & 15 \\
\hline 23 & MRSA & 16 & 14 \\
\hline 24 & MRSA & 16 & 15 \\
\hline 25 & MRSA & 20.5 & 18 \\
\hline 26 & MRSA & 20 & 18 \\
\hline 27 & MRSA & 19 & 17 \\
\hline 28 & MRSA & 17.5 & 16.5 \\
\hline 29 & S. aureus & 16.5 & 15.5 \\
\hline 30 & S. aureus & 21 & 20 \\
\hline 31 & S. aureus & 17 & 16 \\
\hline 32 & S. aureus & 17 & 15 \\
\hline 33 & S. aureus & 20 & 18 \\
\hline 34 & S. aureus & 20 & 18 \\
\hline 35 & S. pyogenes & 19 & 18 \\
\hline 36 & Streptococcus spp & 15 & 13 \\
\hline 37 & Streptococcus spp., viridans group & 14 & 10 \\
\hline
\end{tabular}


Chart.2 Variations in decisive interpretations

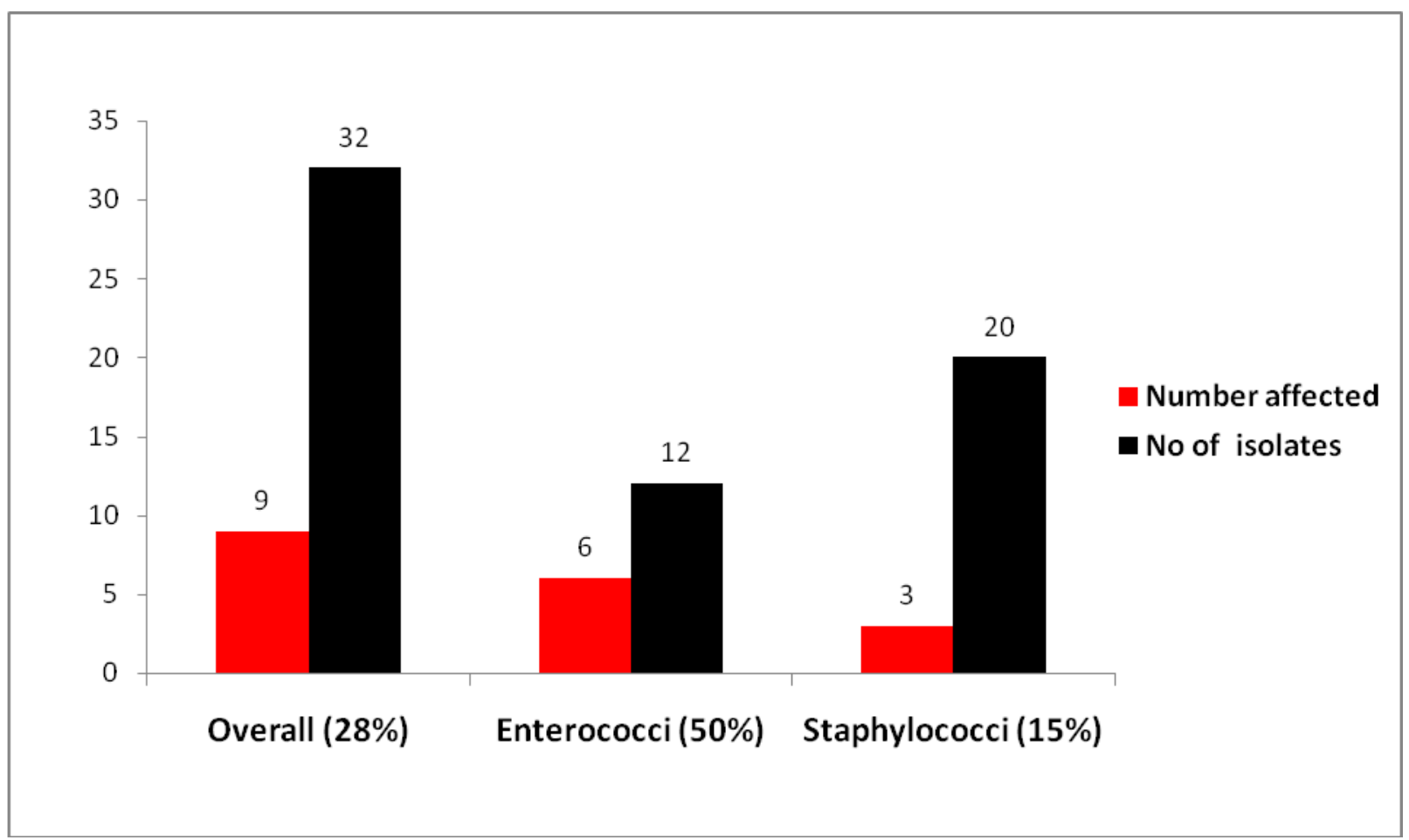

Public commitment to quality is the only way ahead

Public commitment for implementing quality in healthcare has been lacking in our country either because of lack of awareness, laxity thereof, or financial constraints and needs to be addressed (Sinha M et al., 2018). Accreditation by ISO 15189 is not mandatory for clinical laboratories in India and of the estimated 1, 00,000 pathology and diagnostic labs, only about $1000(1 \%)$ are accredited by NABL (NABL 128, 2019). Hence, majority of the clinical laboratories in the country might as well be kept in the darkness of substandard ways of testing and interpreting antibiotic susceptibilities due to unavoidable circumstances, let alone monitoring the quality of the commercially available testing materials. External Quality Assurance schemes (EQAS), when made mandatory for all labs irrespective of accreditation will probably solve this issue. The Laboratorybased surveillance of antimicrobial resistance report of a bi-regional workshop in Chennai (World Health Organization. 2011) has stressed upon the need for improvement in the quality aspects of antimicrobial sensitivity testing while comparing similar scenario in the other countries of the South-East Asian region.

Globally too, not all labs are accredited yet but, are moving towards complete accreditation of quality in the near future. In the review study (T Zima. 2017), the number of accredited medical labs in the Czech Republic has been increasing. The study also quotes that according to the French Law No. 2013-442 of May 30, 2013, all tests in medical laboratories would be accredited by November 1, 2020 and other countries could have similar goals.

The present study concluded, Variation in vancomycin disc content poses significant challenges in routine diagnostic microbiology practice and hence, appropriate makes of discs 
must be chosen to minimize errors and dilemmas in interpretation of susceptibility patterns as per standard guidelines against commonly encountered gram positive cocci. A robust internal quality control system complemented by the general public commitment to adapt to the changing needs is essential for timely identification and correction of possible errors. As change happens over a period of time, the importance of resilient commitment during a transitionary process need not be over-emphasized. In addition, in India, there is an urgent need for a national interpretative reference standard to address the regional specificities of the microbial strains and more importantly in order to aid monitoring its prompt implementation through its easy availability in all modern healthcare settings.

\section{Acknowledgements}

We are grateful to our institutions, colleagues, technical and other staff for their valuable support in bringing out this study.

\section{References}

Adhikari R, Pant ND, Neupane S, Neupane M, Bhattarai R, Bhatta S, Chaudhary R, and Lekhak B. 2017. Detection of Methicillin Resistant Staphylococcus aureus and Determination of Minimum Inhibitory Concentration of Vancomycin for Staphylococcus aureus Isolated from Pus/Wound Swab Samples of the Patients Attending a Tertiary Care Hospital in Kathmandu, Nepal. Hindawi: Canadian Journal of Infectious Diseases and Medical Microbiology, 2191532:1-6.

Ali K, G Omuse, Z Premji and G Revathi. 2016. Comparison of Clinical Laboratory Standards Institute and European Committee on Antimicrobial Susceptibility Testing guidelines for the interpretation of antibiotic susceptibility at a University teaching hospital in Nairobi, Kenya: a crosssectional study. Ann Clin Microbiol Antimicrob, 15(21): 1-7.

Ashtiani MTH, Aboutorabi SK, Mahjoub F, Mamishi S, Asgari F. 2008. Comparison of Disc Diffusion and $\mathrm{E}$ test methods for Antimicrobial Susceptibility Testing of Vancomycin in Coagulase Negative Staphylococcus Isolated from Blood Culture. Iranian Journal of Pathology, 3 (2): 61- 66.

Bhatt Maj P, Bhalla GS, Tandel K, Jindamwar $\mathrm{P}$, Chaudhari $\mathrm{CN}$ and Sahni NGAK. 2015. Antimicrobial Susceptibility Profile of Methicillin-resistant Staphylococcus aureus at a Tertiary Care Centre. Archives of Clinical Microbiology, 6 (3:6): 1-5.

Bora P, Datta P, Gupta V, Singhal L, Chander J. 2018. Characterization and antimicrobial susceptibility of coagulase-negative staphylococci isolated from clinical samples. Journal of Laboratory Physicians, 10(4):41419.

Brown DFJ, Kothari D. 1975. Comparison of antibiotic discs from different sources. J Clinical Pathol., 28:779-83.

Bukhari SZ, Ahmed S, Zia N. 2011. Antimicrobial susceptibility pattern of staphylococcus aureus on clinical isolates and efficacy of laboratory tests to diagnose MRSA: a multi-centre study. J Ayub Med Coll Abbottabad; 23(1): 139-142.

Clinical and Laboratory Standards Institute. 2006. Performance standards for antimicrobial susceptibility testing; sixteenth informational supplement. CLSI document M100-S18.Wayne, PA: Clinical and Laboratory Standards Institute.

Clinical and Laboratory Standards Institute. 2010. Performance standards for 
antimicrobial susceptibility testing; twentieth informational supplement. CLSI document M100-S18.Wayne, PA: Clinical and Laboratory Standards Institute.

Cusack TP, Ashley EA, Ling CL, Roberts T, Turner P, Wangrangsimakul T, Dance DAB. 2019. Time to switch from CLSI to EUCAST? A Southeast Asian perspective. Clinical Microbiology and Infection, 25: 782-785.

El-Said El-Banna T, Sonbol FI, Abd El-Aziz AA, El-Ekhnawy E. 2018. Prevalence and Antimicrobial Susceptibility of Vancomycin Resistant Staphylococci in an Egyptian University Hospital. J Med Microb Diagn, 7(1): 272.

Joshi A, Iyer V, Balasubramaniam U, Kagal A, Bharadwaj R. 2008. Comparison of efficacy of three antibiotic discs commercially available. Indian Journal of Medical Microbiology, 26(2): 1602.

Kiehlbauch JA, Hannett GE, Salfinger M, Archinal W, Monserrat C, and Carlyn C. 2000. Use of the National Committee for Clinical Laboratory Standards Guidelines for Disk Diffusion Susceptibility Testing in New York State Laboratories. Journal of Clinical Microbiology: 3341-3348.

MacKenzie FM, Bruce J, Looveren MVan, Comaglia G, Gould IM, Goossens H, the ARPAC Steering Group. 2006. Antimicrobial susceptibility testing in European hospitals: report from the ARPAC study. Clinical Microbiology and Infection, 12(12) : 1185-1192

Naimi HM, Rasekh H, Noori AZ and MA, Bahaduri. 2017. Determination of antimicrobial susceptibility patterns in Staphylococcus aureus strains recovered from patients at two main health facilities in Kabul, Afghanistan. BMC Infectious Diseases, 17:737(1-7). Nandan TM, Vidyasagar K, Ravikumar R.
2012. Utility of Kirby Bauer disc diffusion method for vancomycin susceptibility testing of staphylococcal isolates: practicability, acceptability, and quality aspects. JEMDS, 1(5):764768.

Procedure for Quality Assurance Scheme for Basic Composite Medical Laboratories (Entry Level). 2019. (NABL 128; Issue No: 02, Issue Date: 17-Sept2019,). Available from: http://www.nabl-india.org [Last accessed on 2019 Nov 10].

Satyanarayana K. 2009. Detection of antimicrobial resistance in common gram negative and gram positive bacteria encountered in infectious diseases - an update. ICMR Bulletin, Vol.39 (1-3): 1-19.

Sharma P, Vishwanath G. Study of vancomycin susceptibility in methicillin-resistant Staphylococcus aureus isolated from clinical samples. 2012. Ann Trop Med and Public Health, 5(3):178-180. [Last accessed: Nov 2, 2019 ] Available from: http://www.atmph.org/text.asp?2012/5/ 3/178/98609

Sinha M, Saini S, Gupta P, Gulati NS, Das A, Kumar A, et al., 2018. Current status and way forward for National Accreditation Board for Testing and Calibration Laboratories Accreditation of Laboratories in Government Organizations. Indian J Pathol Microbiol, 61:461-3.

Thati V, Shivannavar CT \& Gaddad SM. 2011. Vancomycin resistance among methicillin resistant Staphylococcus aureus isolates from intensive care units of tertiary care hospitals in Hyderabad. Indian $\mathbf{J}$ Med Res, 134:704-708.

Wongthong S, Dutchanutouch K, Namsaengkang V, Chanawong A, Wilailuckana C, Lulitanond A. 2015. 
Performance of vancomycin and teicoplanin disk diffusion test in isogenic vancomycin non-susceptible Staphylococcus aureus. J Infect Dev Ctries, 9(2):157-164.

Wootton M, MacGowan AP and Howe RA. 2016. Towards better antimicrobial susceptibility testing: impact of the Journal of Antimicrobial Chemotherapy. J Antimicrob
Chemother 2016. 72: 323-329.

World Health Organization. 2011. Laboratorybased surveillance of antimicrobial resistance. Report of a bi-regional workshop Chennai, India, 21-25 March 2011. [Last accessed on 2019 Nov 10].

Zima T. 2017. Accreditation of medical laboratories-system, process, benefits for labs. J Med Biochem, 36: 231-237.

\section{How to cite this article:}

Nandan, T. M., Abhinandnan T. Kalghatgi, G. Latha and Veena, C. N. 2020. Comparison of Two Commercially Available Vancomycin Discs for Interpreting Susceptibility Patterns among Gram Positive Cocci: Ongoing Challenging Journey in Resource Poor Settings. Int.J.Curr.Microbiol.App.Sci. 9(01): 87-97. doi: https://doi.org/10.20546/ijcmas.2020.901.010 